\title{
Analysis of Ibuprofen and Naproxen Based Two NSAID Candidates with Theoretical DFT Calculations
}

\author{
Nil Ertekin Binbay \\ Dicle University, Vocational School, \\ Department of Electronics, 21280, Diyarbakir, Turkey \\ E-mail: nbinbay@gmail.com
}

\begin{abstract}
Theoretical quantum calculations have been carried out for two new ibuprofen and naproxen based Non-Steroid Anti-inflammatory Drug candidates. DFT (Density Functional Theory) calculations of molecules have been carried out with a general quantum chemistry software package GAMESS (the General Atomic and Molecular Electronic Structure System). Both molecules have been optimised successfully, and IR (Infra-Red) spectra have been obtained via vibrational HESSIAN analyses. The frontier orbitals, HOMO (the Highest Occupied Molecular Orbital) and LUMO (the Lowest Unoccupied Molecular Orbital) have been obtained, and also molecular dynamic descriptors, such as chemical hardness, electronegativity and electrophilicity have been calculated for both molecules. Electrostatic potentials, total molecular electron densities also have been calculated for optimised molecules. All the result has been represented comparatively for both molecules.
\end{abstract}

Keywords: Ibuprofen, Naproxen, DFT, GAMESS.

DOI: $10.7176 / \mathrm{JHMN} / 78-03$

\section{Introduction}

Non-Steroidal Anti-inflammatory Drugs (NSAIDs), and some derivatives of them, have been using for treating pain and inflammatory widely for decades.(Dugowson and Gnanashanmugam 2006; Klasser and Epstein 2005) It is well known that, these drugs have serious gastrointestinal side effects especially on long term use.(Binbay and Ziyadanogullari 2018; Rocha et al. 2001) One of the main strategies to overcome these side effects has been to search for novel drug candidates which obtained by modification of known NSAIDs.(Küçükgüzel et al. 1999; Melnyk et al. 2006; Todeschini et al. 1998; Wu et al. 2007) Here we have worked on two molecules which have been obtained within this perspective in another study, by deriving two well-known NSAIDs ibuprofen and naproxen respectively.(Gökoğlu et al. 2016) First of them is 2-(4-i-Butyl-phenyl)-propionic acid (pyridine-2ylmethylene)-hydrazide, which derived from ibuprofen, and from now on it will be called as AHI shortly. The other one is 2-(6-Methoxy-naphthalen-2-yl)-propionic acid(pyridine-2-ylmethylene)hydrazide, which is derived from naproxen, and from now on it will be called as AHN shortly.(Gökoğlu et al. 2016)

The aimed main goal of these molecules is to create anti-inflammatory effect by binding to the trypsin enzyme, just like the original NSAIDs which they were derived from, namely ibuprofen and naproxen; but of course, with expectation of reduced side effects.(Gökoğlu et al. 2016) As it is pointed out in earlier study, there are strong clues which implies that AHI and AHN are bind trypsin enzyme.(Gökoğlu et al. 2016)

The aim of this study is to contribute to process of understanding of chemical properties that underlies both therapeutic and side effects of $\mathrm{AHI}$ and $\mathrm{AHN}$, by analysing their structural and dynamical properties in detail via DFT calculations.

\section{Material and Method}

\subsection{DFT study}

A general ab-initio quantum chemistry package GAMESS (the General Atomic and Molecular Electronic Structure System) has been used for DFT (Density Functional Theory) calculations.(Gordon and Schmidt 2005; Schmidt et al. 1993) The hybrid Becke, three-parameter, Lee-Yang-Parr, using 
Coulomb Attenuating Method exchange correlation functional (CAM-B3LYP) was used for calculate optimum conformations vibrational states.(Becke 1993, 1996) The basis set for calculations was Dunning/Hay DZ and the polarisation parameter was selected as Dunning.

\subsection{Calculations of chemical descriptors}

The Highest Occupied Molecular Orbitals (HOMOs) and the Lowest Unoccupied Molecular Orbitals (LUMOs) have obtained, and chemical descriptors, like chemical hardness $(\eta)$, electronegativity $(\chi)$, and electrophilicity $(\omega)$, were calculated for each molecule. As it is known, for a system which contains $\mathrm{N}$ electrons and total energy E, chemical hardness $(\eta)$ and electronegativity $(\chi)$ can be defined as (Chaquin 2008):

$$
\begin{aligned}
& \eta=\frac{1}{2}\left(\frac{\partial^{2} \boldsymbol{E}}{\partial \boldsymbol{N}}\right)_{v(r)} \\
& \chi=-\left(\frac{\partial \boldsymbol{E}}{\partial \boldsymbol{N}}\right)_{v(r)}
\end{aligned}
$$

Here $v(r)$ represents the external potential, and $\mathrm{E}$ is the total electronic energy of the $\mathrm{N}$ electron system. For molecules those have closed energy shell, $\chi$ and $\eta$ can be defined also as (Chaquin 2008):

$$
\begin{aligned}
& \chi=\frac{I+A}{2} \\
& \eta=\frac{I-A}{2}
\end{aligned}
$$

Here $\mathrm{I}=-\mathrm{E}_{\mathrm{HOMO}}$ and $\mathrm{A}=-\mathrm{E}_{\mathrm{LUMO}}$. There is also another chemical descriptor, electrophilicity $(\omega)$, which can be defined as (Chaquin 2008):

$$
\omega=\frac{\chi^{2}}{2 \eta}
$$

It is known that there is strong correlation between these descriptors and chemical behaviours of molecules in chemical processes.(Parr and Pearson 1983) For example $E_{\text {Hомо }}$, which describes the energy of highest molecular orbital that contains occupied by electrons, directly relative to the ionisation energy of the molecule, and also solely describes the sensitivity of the molecule upon any electrophilic attack.(Parr and Pearson 1983) On the other hand, energy gap between highest occupied molecular orbital and lowest unoccupied molecular orbital $\left(\mathrm{E}_{\mathrm{LUMO}}-\mathrm{E}_{\mathrm{HOMO}}\right)$ is indicates the measure of activity of the molecule in chemical reactions, and it is directly determines the chemical hardness $(\eta)$ of the molecule.(Pearson 1997) If there is a larger energy gap between these two molecular orbitals, then it is said that this molecule is "chemically harder"; and vice versa. If the gap is smaller, then it is said that the molecule is "chemically softer". Chemically softer molecules are expected to be more active in chemical reactions in general.(Pearson 1997)

\section{Results and Discussion}

The optimised structures of AHI and AHN are given in figure 1 and figure 2 respectively. As it is seen, the structural properties, the distances between atoms, positions of the atoms according to the each other, and bond angels of the atoms are almost same for the atomic groups which ibuprofen and naproxen bonded to, in both molecules

The calculated electrostatic potentials of AHI and AHN are given in figure 3 and figure 4 respectively. As it is seen, although both of the electrostatic potentials have similar structures in general, there is an apparent distinction between electrostatic potentials of ibuprofen and naproxen parts of the molecules. It is seen that, the oxygen atom that placed just before methyl group in AHN, have created a difference in electrostatic potential of the molecule by attracting electrons close to it. 


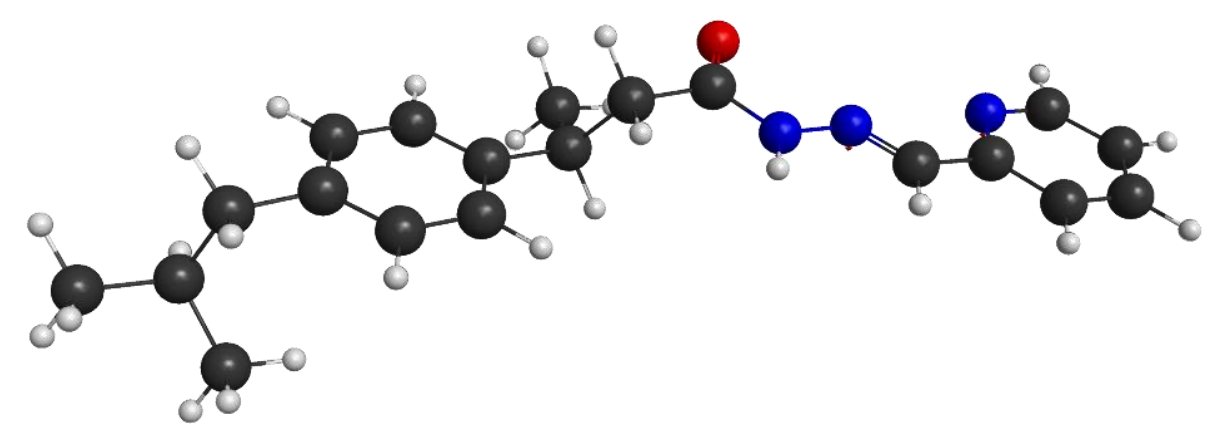

Figure 1. Optimised structure of AHI.

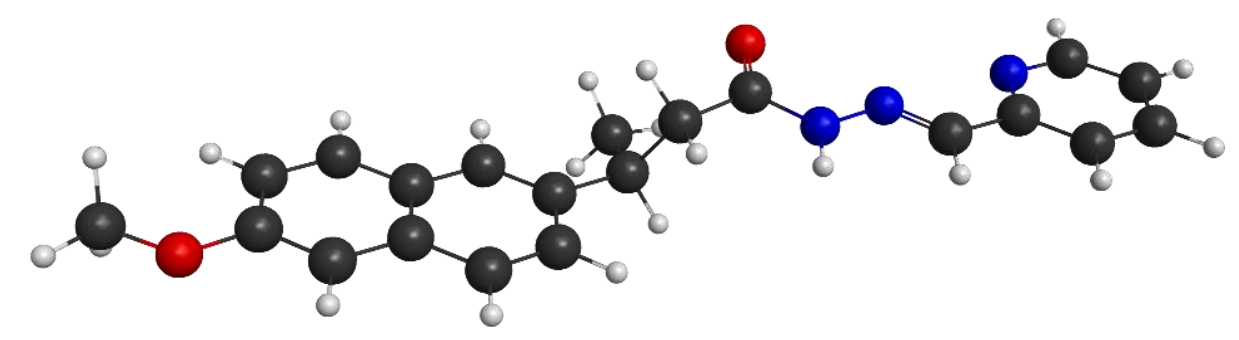

Figure 2. Optimised structure of AHN.



Figure 3. Molecular electrostatic potential of AHI. 


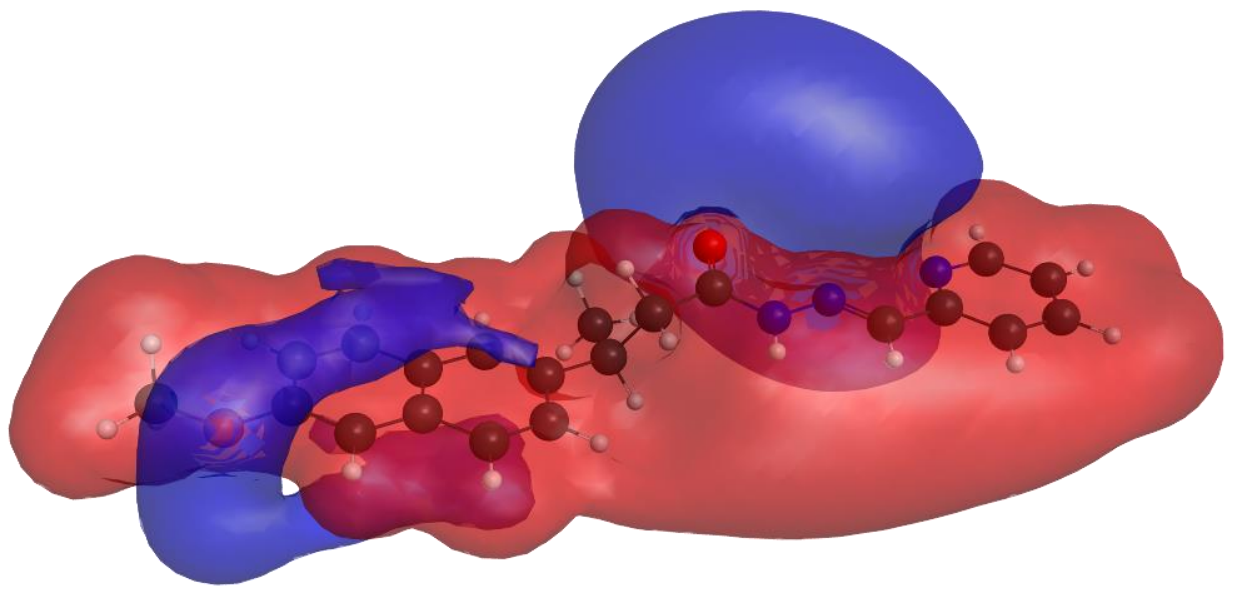

Figure 4. Molecular electrostatic potential of AHN.

The total electron densities of the AHI and AHN are given in figure 5 and figure 6 respectively. As it is seen, the electron densities of the atomic group which ibuprofen and naproxen are bonded to, are similar for both molecules. But it can be seen in figure 6 that, as the oxygen that placed just after the methyl group in AHN, attracts more electrons to itself, there is apparently more electronic density near it. Another point which can be noticed in figure 6 is that, the methyl group which placed at the end of the AHN has less electronic density (ie. more positive) than the same two methyl group of the AHN which placed at the end of the molecule. That means the oxygen-methyl area of AHN is more polarised than AHI. As polarisation is one of the most important factors about intermolecular interactions, it is expected that AHN will be chemically more active than AHI.

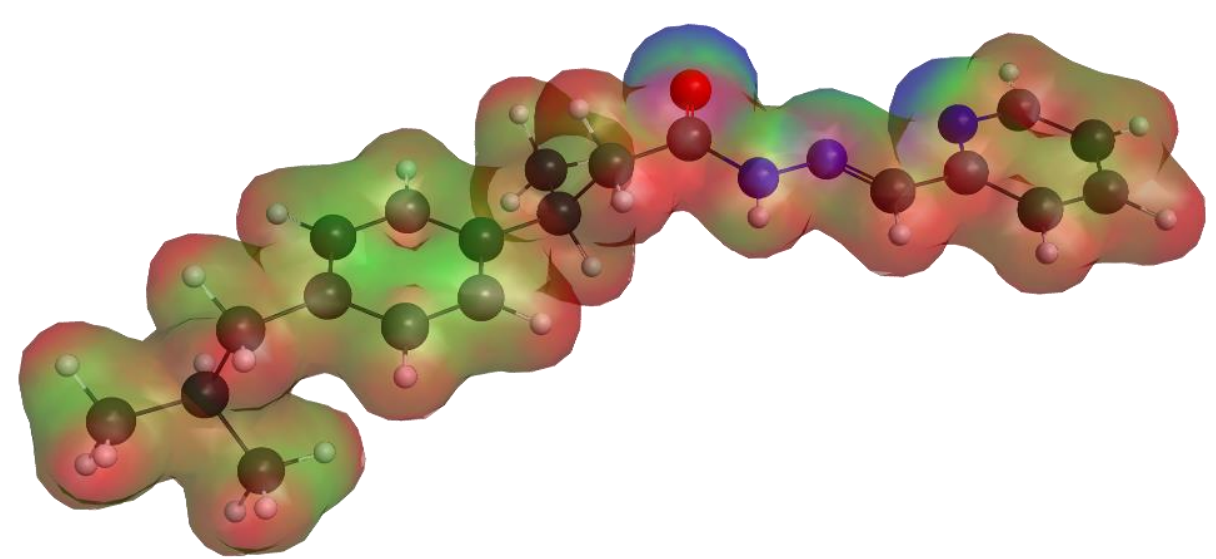

Figure 5. Total electron density of AHI. 


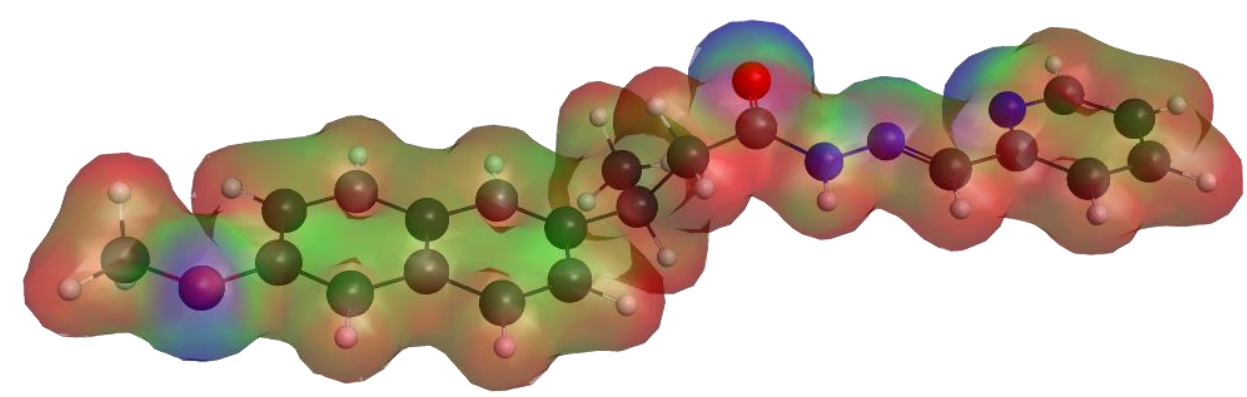

Figure 6. Total electron density of AHN.

The frontier orbitals (HOMOs and LUMOs) of AHI and AHN and the energy gaps between them is given in figure 7 and figure 8 respectively. As it is seen, the energy level of AHI's LUMO is higher than AHN's LUMO, and also the energy level of AHI's HOMO is lower than AHN's HOMO. Therefore, AHN has a larger energy gap than AHI, between frontier orbitals HOMO and LUMO. Hence, within the perspective that the energy levels of frontier orbitals and the energy gap between them are important parameters for determining the chemical activeness of molecules; it is expected that AHN can be more active than AHI chemically.

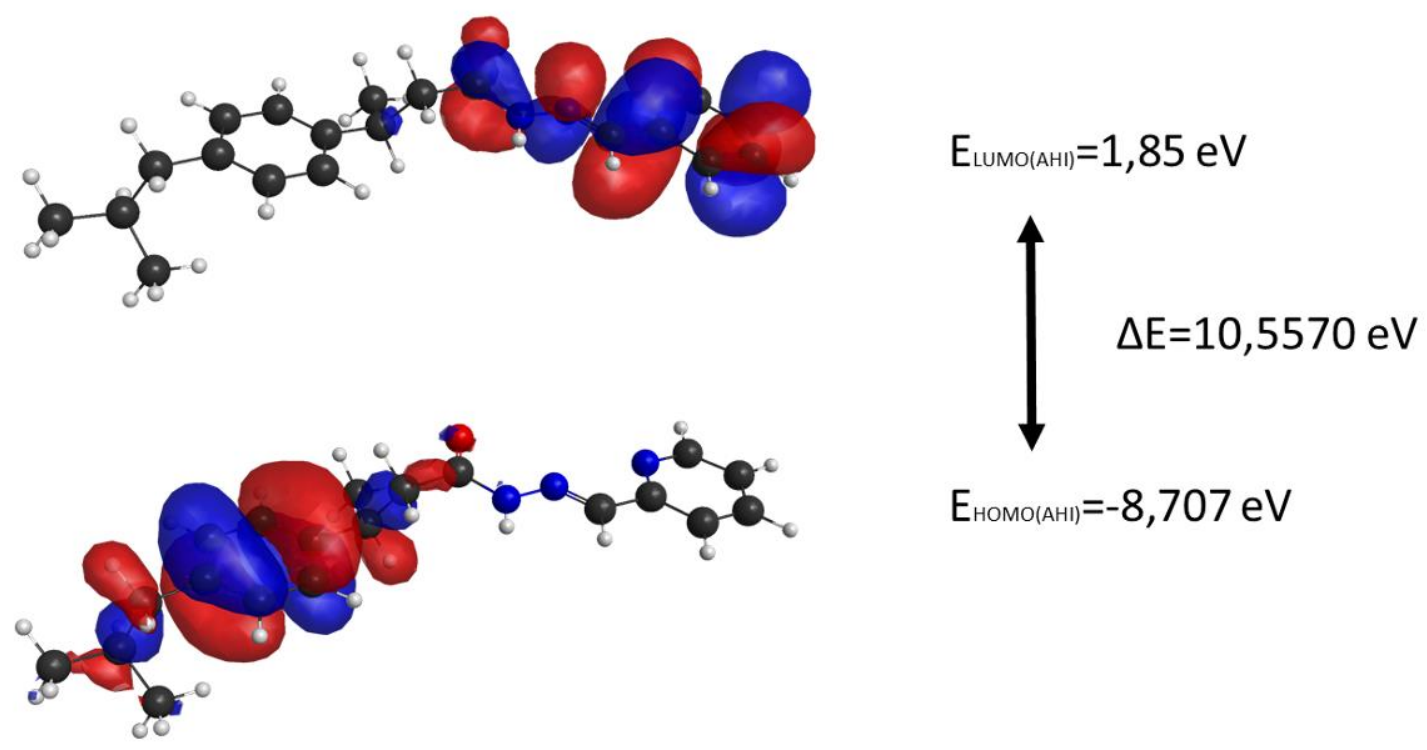

Figure 7. HOMO and LUMO of AHI. 




$E_{\text {Luмо(AHN) }}=1,768 \mathrm{eV}$
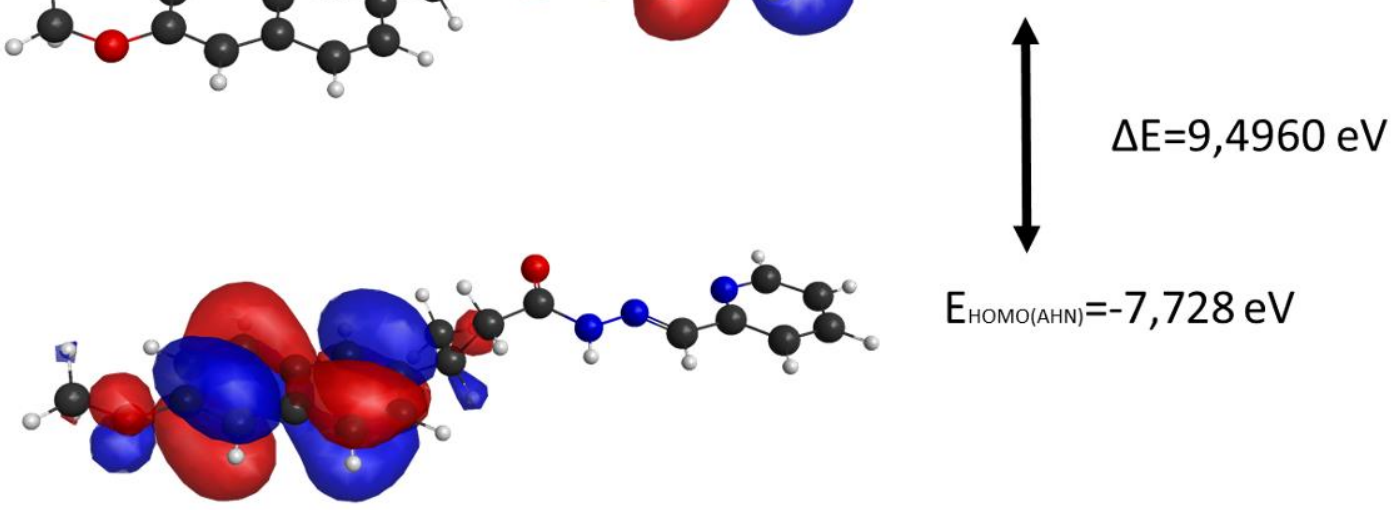

Figure 8. HOMO and LUMO of AHN.

Another point that can be noticed is that, LUMOs of the both molecules are seem to be constructed from combination of same atomic orbitals and structure of them looking very same. On the other hand, it is seen that, overall differences of molecules can change the energy level of the molecular orbital LUMO, by without changing its structure or shape at all. For AHI $\mathrm{E}_{\mathrm{LUMO}}$ is $1.850 \mathrm{eV}$, while for AHN $\mathrm{E}_{\mathrm{LUMO}}$ is $1.768 \mathrm{eV}$.

Calculated Infra-Red (IR) spectra of AHI and AHN are given in figure 9 and figure 10 respectively. As it is seen in figure 9, the most intense vibration of the AHI's IR spectrum at $1245.88 \mathrm{~Hz}$, that mainly occurs between oxygen and the carbon which is double bonded to oxygen. On the other hand, as it is seen in figure 10, most intense molecular vibration in AHN's IR spectrum at $1701.16 \mathrm{~Hz}$, which mainly occurs between the nitrogen atoms that bonded to each other.

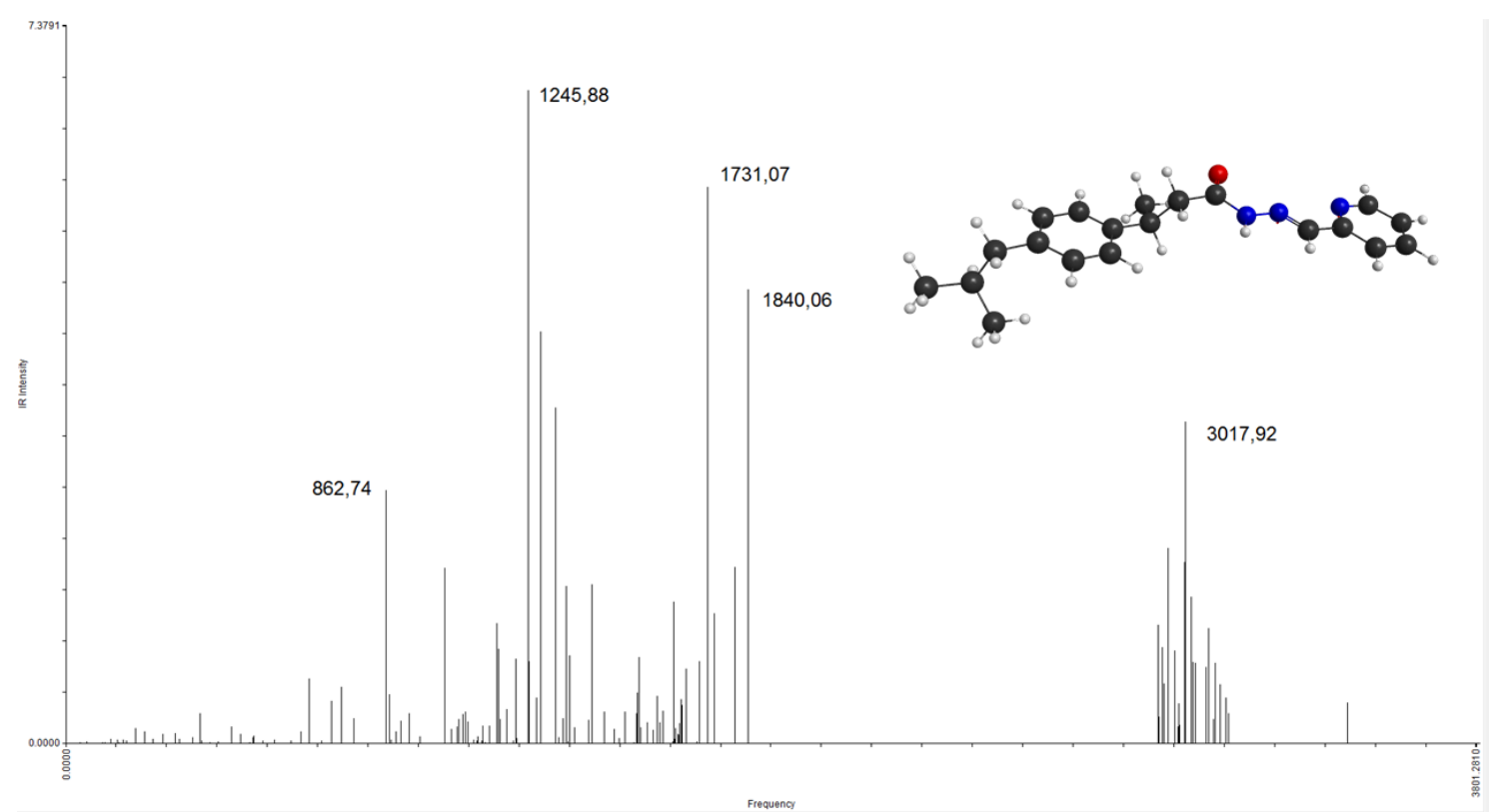

Figure 9. IR spectrum of AHI. 




Figure 10. IR spectrum of AHN.

The calculated chemical descriptors for AHI and AHN, HOMOs, LUMOs, chemical hardness values $(\eta)$, electronegativities $(\chi)$ and electrophilicities $(\omega)$ are given altogether in table 1 . As it is seen, the energy level of the highest occupied molecular orbital of $\mathrm{AHI}\left(\mathrm{E}_{\mathrm{HOMO} / \mathrm{AHI}}\right)$ is lower than same molecular orbital for AHN ( $\left.\mathrm{E}_{\mathrm{HOMO} / \mathrm{AHN}}\right)$. Also, the energy level of the lowest unoccupied molecular orbital of AHI $\left(\mathrm{E}_{\mathrm{LUMO} / \mathrm{AHI}}\right)$ is higher than the same molecular orbital for $\mathrm{AHN}\left(\mathrm{E}_{\mathrm{LUMO} / \mathrm{AHN}}\right)$. Therefore, $\mathrm{AHI}$ has larger energy gap than $\mathrm{AHN}$, between its frontier orbitals $\left(\left(\mathrm{E}_{\mathrm{LUMO} / \mathrm{AHI}}-\mathrm{E}_{\mathrm{HOMO} / \mathrm{AHI}}\right)>\left(\mathrm{E}_{\mathrm{LUMO} / \mathrm{AHN}}-\mathrm{E}_{\mathrm{HOMO} / \mathrm{AHN}}\right)\right)$. Hence, AHI is chemically harder than AHN, in other words, AHI is more stable than AHN. But this is not a preferred property from perspective of chemical activity in reactions. As it can also be seen in table 1, AHI is both more electrophilic and electronegative than AHN. As AHN is chemically softer than AHI $\left(\eta_{\mathrm{AHN}}<\eta_{\mathrm{AHI}}\right)$, it is expected that AHN presents more active behaviour in chemical reactions. This is compatible with literature.(Gökoğlu et al. 2016)

Table 1. Calculated chemical descriptors for AHI and AHN

\begin{tabular}{|l|l|l|l|l|l|l|}
\hline Molecule & $\begin{array}{c}\mathbf{E}(\mathrm{HOMO}) \\
(\mathbf{e V})\end{array}$ & $\begin{array}{c}\mathbf{E}(\mathbf{L U M O}) \\
(\mathbf{e V})\end{array}$ & $\begin{array}{c}\Delta \mathbf{E}= \\
\mathbf{E}_{(\mathrm{LUMO})}-\mathbf{E}_{(\mathrm{HOMO})} \\
(\mathbf{e V})\end{array}$ & $\begin{array}{c}\boldsymbol{\eta} \\
(\text { chemical hardness) } \\
(\mathbf{e V})\end{array}$ & $\begin{array}{c}\boldsymbol{1} \\
(\text { electronegativity) } \\
(\mathbf{e V})\end{array}$ & $\begin{array}{c}\omega \\
(\text { electrophilicity) } \\
(\mathbf{e V})\end{array}$ \\
\hline AHI & -8.707 & 1.850 & 10.5570 & 5.2785 & 3.4285 & 1.1134 \\
\hline AHN & -7.728 & 1.768 & 9.4960 & 4.7480 & 2.9800 & 0.9352 \\
\hline
\end{tabular}

The calculated chemical descriptors for AHI and AHN; energy level of HOMOs and LUMOs, chemical hardness values $(\eta)$, electronegativities $(\chi)$ and electrophilicities $(\omega)$ are given altogether.

\section{Conclusion}

Here a computational study, includes DFT calculations and theoretical analysis of two NSAID candidate molecule which derived from ibuprofen and naproxen respectively, has been completed and presented successfully. Among these two molecules, AHN, which is derived from naproxen, is found to be potentially more active than AHI, which is derived from ibuprofen. Finally, it is concluded that, in the light of biological importance of acylhydrazone derivatives due to their anti-microbial (Loncle et al. 2004; Papakonstantinou-Garoufalias et al. 2002; Vicini et al. 2002), anti-convulsant (Küçükgüzel et al. 2003), and anti-tuberculosis (Patole et al. 2003) effects, both of AHN and AHI has strong potentials as candidates of NSAIDs with reduced side effects. 


\section{References}

Becke, Axel D. 1993. 'Density-functional Thermochemistry. III. The Role of Exact Exchange'. The Journal of Chemical Physics 98(7):5648-52.

Becke, Axel D. 1996. 'Density-functional Thermochemistry. IV. A New Dynamical Correlation Functional and Implications for Exact-exchange Mixing'. The Journal of Chemical Physics 104(3):1040-46.

Binbay, Nil E., and Berrin Ziyadanogullari. 2018. 'Comparison of Binding Properties of a Novel Non-Steroidal Anti-Inflammatory Agent and Ibuprofen to Cyclooxygenase-1 and Cyclooxygenase-2 Enzymes by Saturation Transfer Difference Nuclear Magnetic Resonance'. Canadian Journal of Physics 96(7):693-99.

Chaquin, Patrick. 2008. 'Absolute Electronegativity and Hardness: An Analogy with Classical Electrostatics Suggests an Interpretation of the Parr "Electrophilicity Index" as a "Global Energy Index" Leading to the "Minimum Electrophilicity Principle"". Chemical Physics Letters 458(1-3):231-34.

Dugowson, Carin E., and Priya Gnanashanmugam. 2006. 'Nonsteroidal Anti-Inflammatory Drugs'. Physical Medicine and Rehabilitation Clinics of North America 17(2):347-54.

Gökoğlu, Elmas, Esra Yılmaz, Esra Gökoğlu, and Ayşe Uzgören Baran. 2016. 'Study of Binding Properties Between Two New Ibuprofen and Naproxen Based Acyl Hydrazone Derivatives and Trypsin'. Journal of Fluorescence 26(1):113-19.

Gordon, Mark S., and Michael W. Schmidt. 2005. 'Advances in Electronic Structure Theory'. Pp. 1167-89 in Theory and Applications of Computational Chemistry. Elsevier.

Klasser, Gary D., and Joel Epstein. 2005. 'Nonsteroidal Anti-Inflammatory Drugs: Confusion, Controversy and Dental Implications'. Journal (Canadian Dental Association) 71(8):575-80.

Küçükgüzel, S. Güniz, Adil Mazi, Fikrettin Sahin, Suzan Öztürk, and James Stables. 2003. 'Synthesis and Biological Activities of Diflunisal Hydrazide-Hydrazones'. European Journal of Medicinal Chemistry 38(11-12):1005-13.

Küçükgüzel, Ş. Güniz, Sevim Rollas, Ilkay Küçükgüzel, and Muammer Kiraz. 1999. 'Synthesis and Antimycobacterial Activity of Some Coupling Products from 4-Aminobenzoic Acid Hydrazones'. European Journal of Medicinal Chemistry 34(12):1093-1100.

Loncle, Céline, Jean Michel Brunel, Nicolas Vidal, Michel Dherbomez, and Yves Letourneux. 2004. 'Synthesis and Antifungal Activity of Cholesterol-Hydrazone Derivatives'. European Journal of Medicinal Chemistry 39(12):1067-71.

Melnyk, Patricia, Virginie Leroux, Christian Sergheraert, and Philippe Grellier. 2006. 'Design, Synthesis and in Vitro Antimalarial Activity of an Acylhydrazone Library'. Bioorganic \& Medicinal Chemistry Letters 16(1):31-35.

Papakonstantinou-Garoufalias, S., N. Pouli, P. Marakos, and A. Chytyroglou-Ladas. 2002. 'Synthesis Antimicrobial and Antifungal Activity of Some New 3-Substituted Derivatives of 4(2,4-Dichlorophenyl)-5-Adamantyl-1H-1,2,4-Triazole'. Il Farmaco 57(12):973-77.

Parr, Robert G., and Ralph G. Pearson. 1983. 'Absolute Hardness: Companion Parameter to Absolute Electronegativity'. Journal of the American Chemical Society 105(26):7512-16. 
Patole, Jayendra, Uday Sandbhor, Subhash Padhye, Dileep N. Deobagkar, Christopher E. Anson, and Annie Powell. 2003. 'Structural Chemistry and In Vitro Antitubercular Activity of Acetylpyridine Benzoyl Hydrazone and Its Copper Complex against Mycobacterium Smegmatis'. Bioorganic \& Medicinal Chemistry Letters 13(1):51-55.

Pearson, Ralph G. 1997. Chemical Hardness: Applications from Molecules to Solids. Weinheim: Wiley-VCH.

Rocha, G. M., L. F. Michea, E. M. Peters, M. Kirby, Y. Xu, D. R. Ferguson, and M. B. Burg. 2001. 'Direct Toxicity of Nonsteroidal Antiinflammatory Drugs for Renal Medullary Cells'. Proceedings of the National Academy of Sciences 98(9):5317-22.

Schmidt, Michael W., Kim K. Baldridge, Jerry A. Boatz, Steven T. Elbert, Mark S. Gordon, Jan H. Jensen, Shiro Koseki, Nikita Matsunaga, Kiet A. Nguyen, Shujun Su, Theresa L. Windus, Michel Dupuis, and John A. Montgomery. 1993. 'General Atomic and Molecular Electronic Structure System'. Journal of Computational Chemistry 14(11):1347-63.

Todeschini, Adriane R., Ana Luisa P. de Miranda, Kelly Christine M. da Silva, Sergio C. Parrini, and Eliezer J. Barreiro. 1998. 'Synthesis and Evaluation of Analgesic, Antiinflammatory and Antiplatelet Properties of New 2-Pyridylarylhydrazone Derivatives'. European Journal of Medicinal Chemistry 33(3):189-99.

Vicini, Paola, Franca Zani, Pietro Cozzini, and Irini Doytchinova. 2002. 'Hydrazones of 1,2Benzisothiazole Hydrazides: Synthesis, Antimicrobial Activity and QSAR Investigations'. European Journal of Medicinal Chemistry 37(7):553-64.

Wu, La-Mei, Han-Bing Teng, Xian-Bing Ke, Wen-Jin Xu, Jiang-Tao Su, Shu-Cai Liang, and XianMing Hu. 2007. 'Copper(II) Complexes of Salicylaldehyde Hydrazones: Synthesis, Structure, and DNA Interaction’. Chemistry \& Biodiversity 4(9):2198-2209. 